\title{
DESIGNING OF COUMARIN DERIVATIVES AS SQUALENE SYNTHASE INHIBITORS
}

\section{FIROZ MV ${ }^{1}$, VISHWANATHAN BALASUBRAMANYA IYER ${ }^{2}$, VISHAL GUPTA N ${ }^{1}$, GOWDA DV ${ }^{1 *}$, GURUPADAYYA BM² AJMAL KP ${ }^{2}$, PRAVEEN SIVADASU ${ }^{1}$}

${ }^{1}$ Department of Pharmaceutics, JSS College of Pharmacy, Sri Shivarathreeshwara Nagara, Mysuru, JSS Academy of Higher Education and Research, JSS Medical Institutions Campus, Sri Shivarathreeshwara Nagara, Mysuru - 570 015, Karnataka, India. ${ }^{2}$ Department of Pharmaceutical Chemistry, JSS College of Pharmacy, Sri Shivarathreeshwara Nagara, Mysuru, JSS Academy of Higher Education and Research, JSS Medical Institutions Campus, Sri Shivarathreeshwara Nagara, Mysuru - 570 015, Karnataka, India. Email: dvgowda@jssuni.edu.in

Received: 02 May 2018, Revised and Accepted: 14 June 2018

\section{ABSTRACT}

Objective: The importance of this research work is to design a library of novel coumarin derivatives by docking evaluation of the designed coumarin derivatives as squalene synthase inhibitor

Methods: The three-dimensional structure of designed molecules of squalene synthase inhibitors was collected from Protein Data Bank. The designed molecules were docked onto the enzymes that are squalene synthase inhibitor - 3WCM, 3WCJ, and 3Q2Z protein using SYBYL-X 2.1. Using a standard protocol, the protein was subjected to minimization and protomol generation.

Results: By this method, we visualized the possible binding and also estimated the protein interactions with our intended coumarin library, using SYBYL-X 2.1 software. Into the active site of the selected enzymes, all the 20 coumarins were docked and then the docking scores revealed that the compounds possess high affinity toward the selected enzymes.

Conclusion: With the help of virtual evaluation, we have elaborated a fast synthetically accessible coumarin-based compounds, and it is an advanced and original scaffold in the area of probable human squalene synthase inhibitors. Some of the developed compounds show better binding property than ligand, and in 3q2Z, the compound $5 \mathrm{~d}$ shows better binding property than the protein. Furthermore, $6 \mathrm{~g}$ and $6 \mathrm{c}$ have good binding property. In 3 WCM, the compound $6 \mathrm{f}$ has better property. In $3 \mathrm{WCJ}$, the compounds $6 \mathrm{~g}$ and $6 \mathrm{f}$ show better binding property than the protein.

Keywords: Coumarin derivatives, Squalene synthase inhibitors, Molecular docking, Docking score.

(C) 2018 The Authors. Published by Innovare Academic Sciences Pvt Ltd. This is an open access article under the CC BY license (http://creativecommons. org/licenses/by/4. 0/) DOI: http://dx.doi.org/10.22159/ajpcr.2018.v11i10.27044

\section{INTRODUCTION}

The main intention of this research work is to design a library of novel coumarin derivatives by docking evaluation of the designed coumarin derivatives as squalene synthase inhibitor. Coumarins are the compounds which can be found in different levels all over the plant kingdom. Some significant coumarins were derived from several microorganisms such as from Streptomyces species [1]. Dyslipidemia is a main risk factor in the occurrence of coronary heart disease (CHD). In case of CHD, dyslipidemia is a major risk factor. According to the World Health Organization, there are around 4 million deaths per year due to the heart diseases which is caused by dyslipidemia [2,3].

Dyslipidemia leads to elevation of total cholesterol, low-density lipoproteins, high-density lipoprotein, and triglycerides. If the elevation of cholesterol occured due to lipoprotein then it is termed as hyperlipidemia, whereas elevation is due to triglycerides, low-density lipoprotein and high-density lipoprotein are called as atherogenic dyslipidemia [2,3].

With the sudden and stark increase in population growth rates the cardiovascular disease, it got affected the developing countries like India. By 2020, about 2.6 million Indians are estimated to die due to coronary heart failure. Among Indians, it has been shown that it occurs too early at least a period of 10 years or two earlier than their counterparts in developed countries [4].

\section{Docking}

Using docking method, we can find the favored orientation of one molecule to a second molecule when bound to each other to form a stable complex. It is commonly used to forecast the binding orientation of small drug molecule to their protein targets to predict the affinity and action of the small drug molecule. Thus, docking plays a significant role in the rational drug design. The aim of docking is to find the threedimensional (3D structure of the macromolecular complex of interest as it would happen in a living organism. Candidate structures which are potential can have produced by the process of docking [5].

\section{Types of docking studies}

- Protein-protein docking

- Protein-ligand docking

- $\quad$ Other docking programs: FlexX, GOLD, Hammerhead, FLOG [4].

\section{MATERIALS AND METHODS}

\section{Molecular docking studies}

SYBYL-X 2.1 software has used to carry out docking studies. This software for docking program helps to gain deep understanding of binding mode of the intended compounds and explains the effect of structural changes on the chosen protein in the direction of their inhibitory activities. The flow diagram for structure-based drug design is shown in Fig. 1. Crystallographic data of the selected enzyme were downloaded and were used for docking studies. In the case of protein and enzymes, the X-ray crystal structure was described and achieved an acceptance with X-ray crystal structure was achieved with the help of hydrogen bond interaction of amino acid residues [6]. Using SYBYL-X 2.1 , the protein has been developed in the basis of preparation module in the software. By this, the bond orders have been allocated, water and other residues have been removed, $\mathrm{H}$ atoms have added, and the 


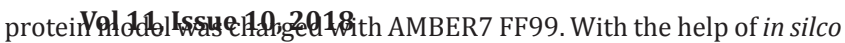
screening, coumarin-based compounds have developed which are fast synthetically accessible compounds [7]. It is a featuring new method in the case of potential human squalene synthase inhibitors. Especially, we have designed a set of compounds from a common scaffold having various groups attached to the coumarin ring, by considering both the synthetic accessibility and affinity with ligand binding domain of squalene synthase [8]. A brief description about structure-based design is depicted in Fig. 1.

\section{Protein minimization}

The structure of human squalene synthase was acquired from the Protein Data Bank (PDB Code: 3WCM, 3WCJ, and 3Q2Z). Graphical representation of 3Q2Z, 3WCJ, and 3WCM is shown in Figs. 2-4, respectively. At 2.38A resolution, this structure is resolved. We accomplished the minimization of human squalene synthase with a template using the prepare protein module in SYBYL [9]. The ligand substructure was extracted, and all water molecules were separated. Explicit hydrogens were added to the protein, and protein model was charged with AMBER7 FF99 and ligand using Gasteiger-Marsili charges. Relaxation of the loop region and energy minimization was accomplished using 300 iterations with AMBER7 FF99 as force field [10].

\section{Protomol generation}

Receptor active binding site (protomol) was generated around the cocrystallized ligands in SYBYL X.2.1 molecular modeling software using default parameters. Ligand was extracted from the protein. The active site was generated to fit the ligands similar in size to cocrystallized ligands. The cocrystallized ligands of 3WCJ and 3WCM are shown in Figs. 5-7, respectively [11].

\section{Preparation of ligands}

The ligand compound was designed using SYBYL software. Here, energy minimization was done using the software and preparation of ligand

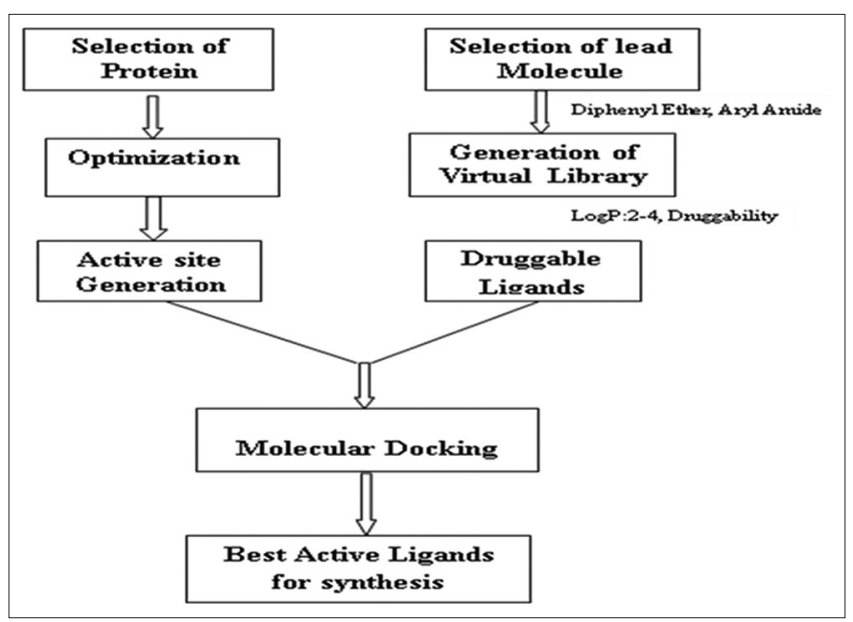

Fig. 1: Flow diagram for structure based drug design

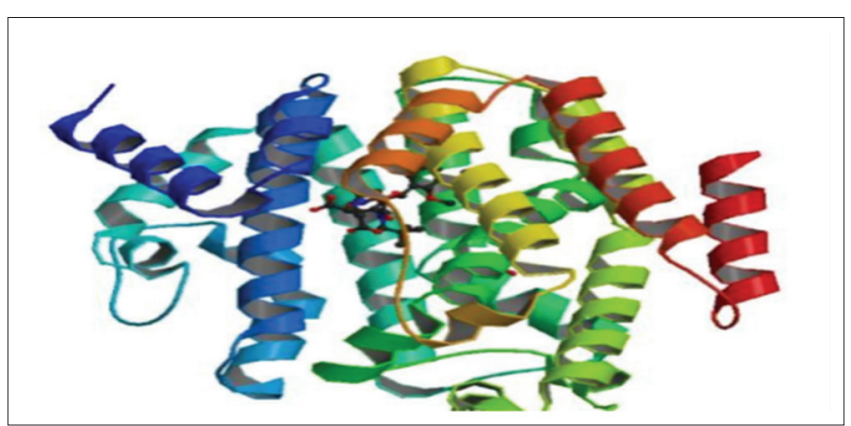

Fig. 2: Graphical representation of 3Q2Z

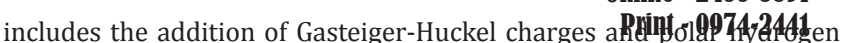
[12]. A number of 20 coumarin derivative were used in the study, which were designed by in silco virtual screening. The library of compounds is listed in Table 1. 20 ligands were drawn in Chem draw. Ligand preparation is a beneficial of SYBYL software suit that links the tools for creating 3D structures from 2D, searching for tautomers and performing a geometry minimization of ligands. The energy minimization of the ligands was performed using minimize module of SYBYL with default parameters and applying molecular mechanics force fields [13].

\section{Docking of ligands}

SYBYL docking suite was helped for this study. The SYBYL docking processes carry out a set of graded examinations for positions of achievable ligand affinity within the binding site of a receptor. A rough arrangement and counting process are applied during the early examination step, come after torsional energy optimization on optimized potentials for liquid simulation-AA non-bonded potential energy grid for generating potential candidate poses. Selection of the final docked pose is done using a total score, which is a model energy function that joins the empirical and force field-based terms [14].

\section{RESULTS AND DISCUSSION}

\section{Results of molecular docking}

The method was used to visualize the probable binding and also to learn and estimate the protein interactions with our intended coumarin library. Using SYBYL-X 2.1 software, the docking study was completed. In which, into the active site of the selected enzymes, all the 20 coumarins were docked. From the Brookhaven, PDB has downloaded the crystal structure of the enzyme along with its cocrystallized ligand and was used for docking studies.

\section{Molecular docking studies on squalene synthase (3WCM)}

The docking study of the designed substituted coumarin onto the active site of the enzyme squalene synthase (PDB: $3 W C M$ ) was encouraging (Fig. 8). The cocrystallized ligand ER4 ((3R)-3-\{[2-benzyl-6-(3methoxypropoxy) pyridin- 3-yl] ethynyl\}-1-azabicyclo [2.2.2] octan-3ol) showed a score of $10.0858 \mathrm{Kcal} / \mathrm{M}$ with the interactions with the all-around residues Leu211A, lle58A, Leu76A, Leu183A, Val179A, and Tyr73A strongly contributed to the stabilization.

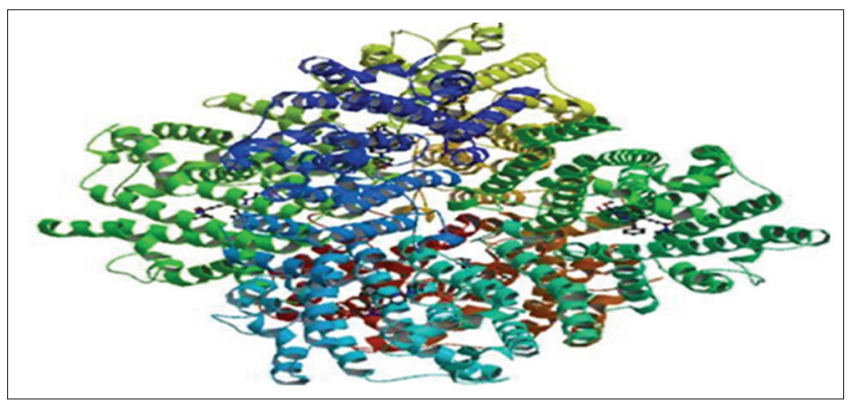

Fig. 3: Graphical representation of 3WC]

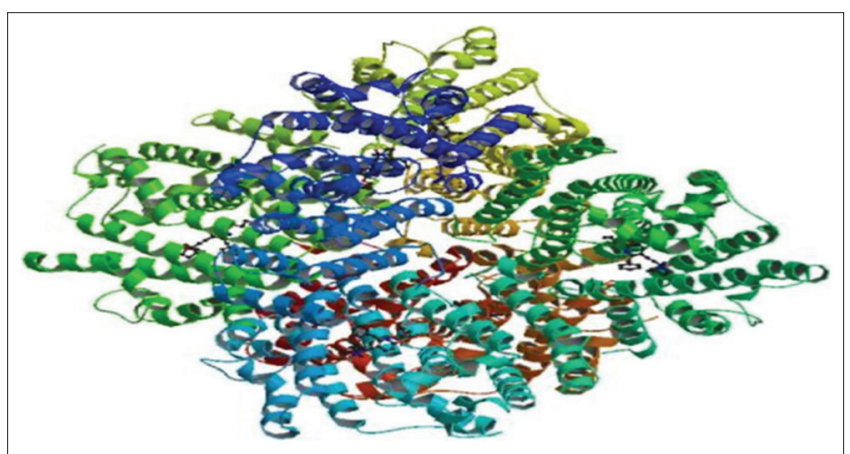

Fig. 4: Graphical representation of 3 WCM 


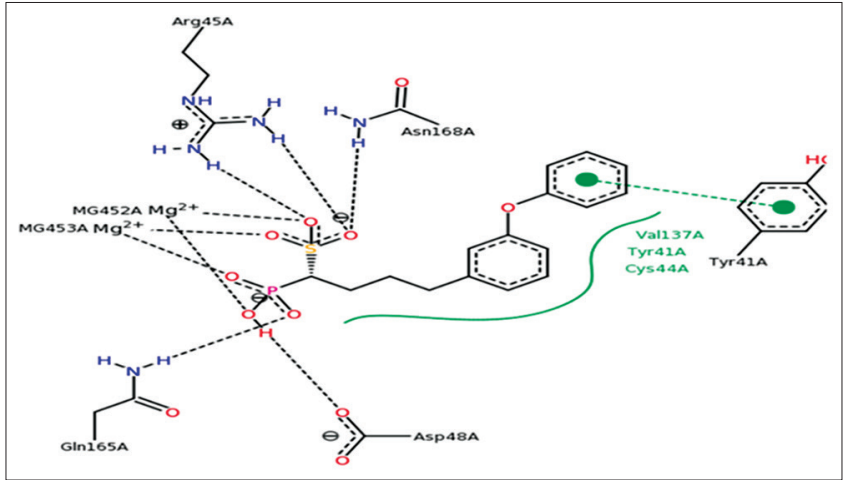

Fig. 5: Cocrystallized ligands of 3WCJ

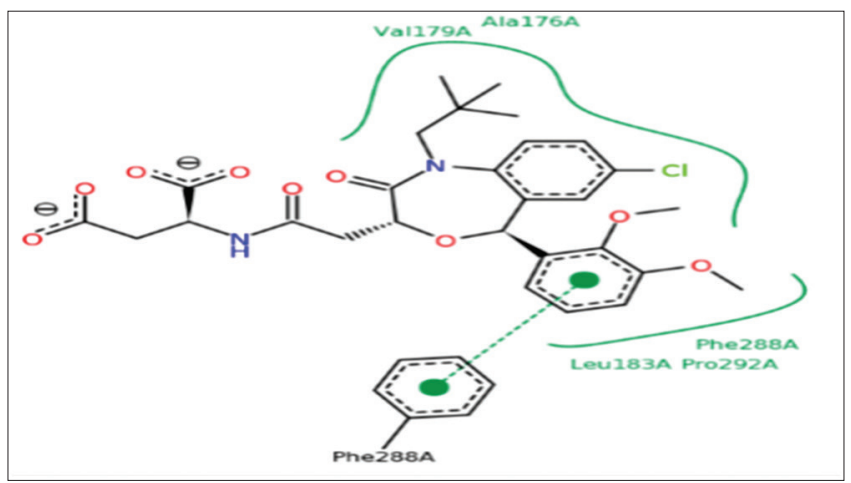

Fig. 6: Cocrystallized ligands of $3 Q 2 Z$

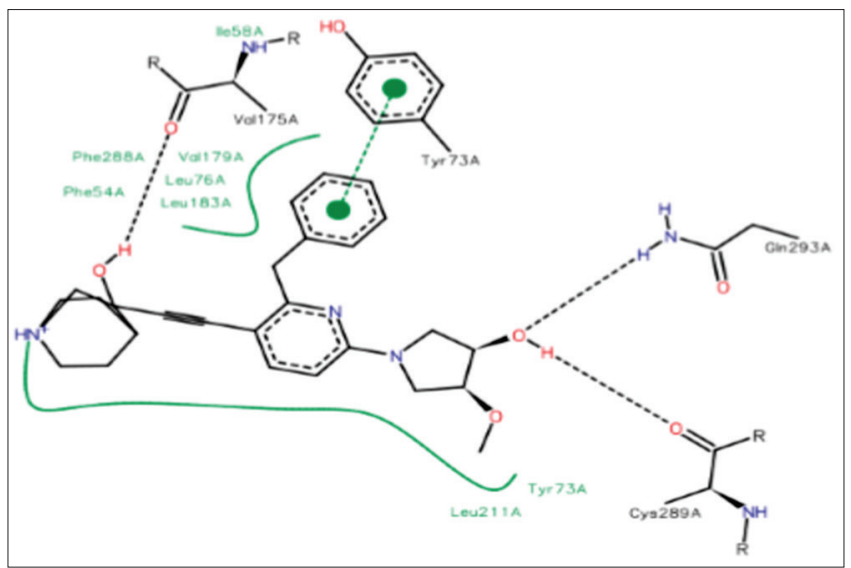

Fig. 7: Cocrystallized ligands of 3WCM

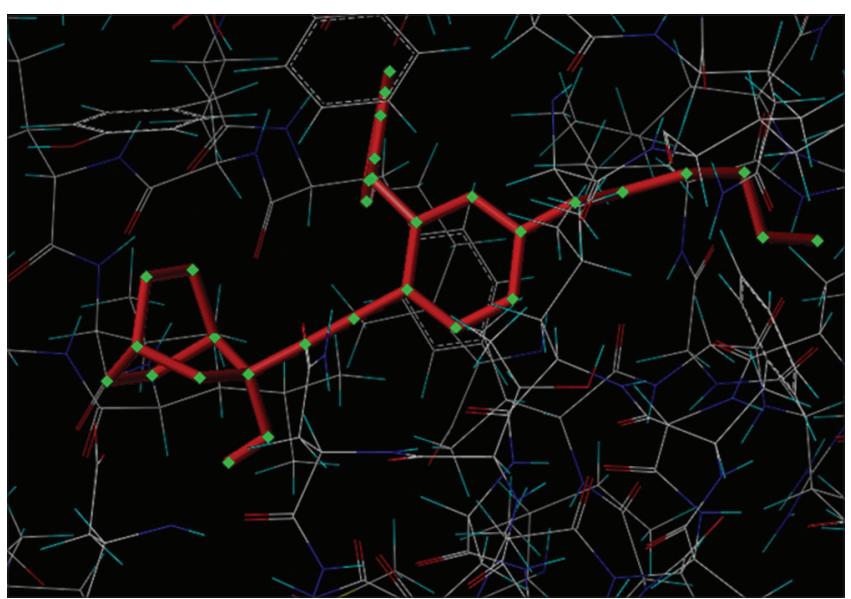

Fig. 8: Active binding in 3WCM

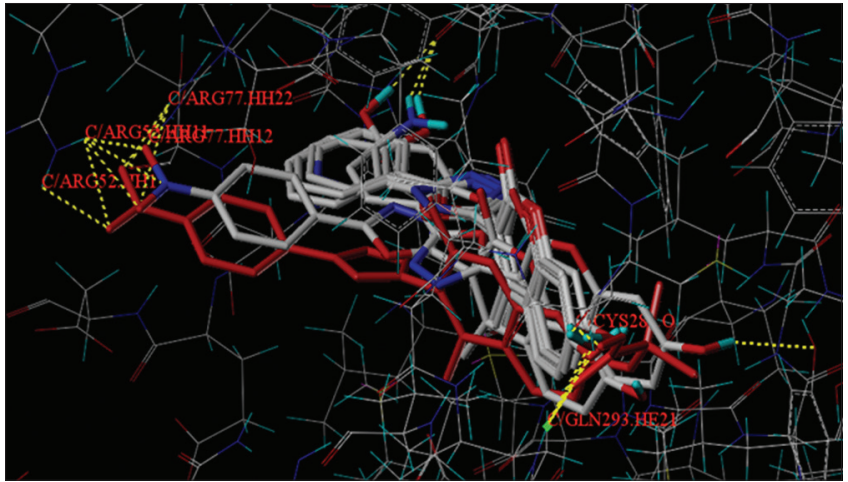

Fig. 9: Conformationally analyzed and energy minimized structures aligned against common atoms interacting with protomol 3WCM

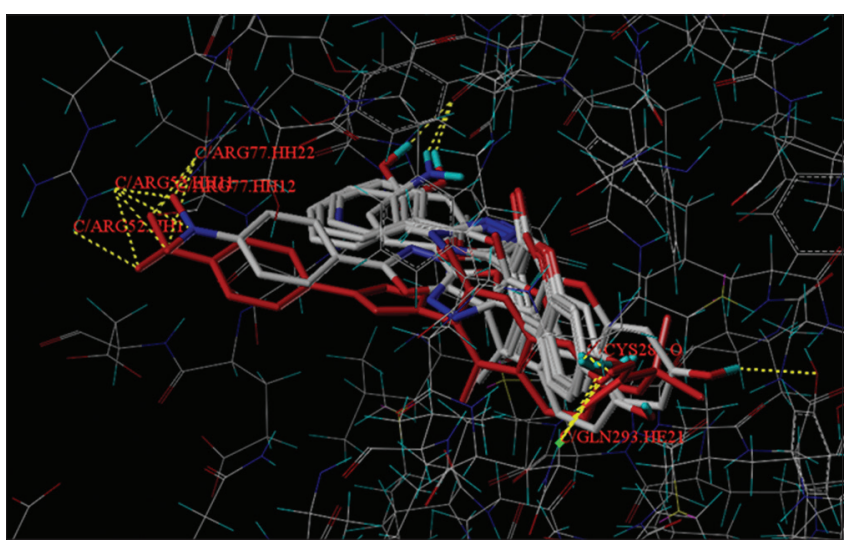

Fig. 10: Docking of 5 series with 3WCM.pdb

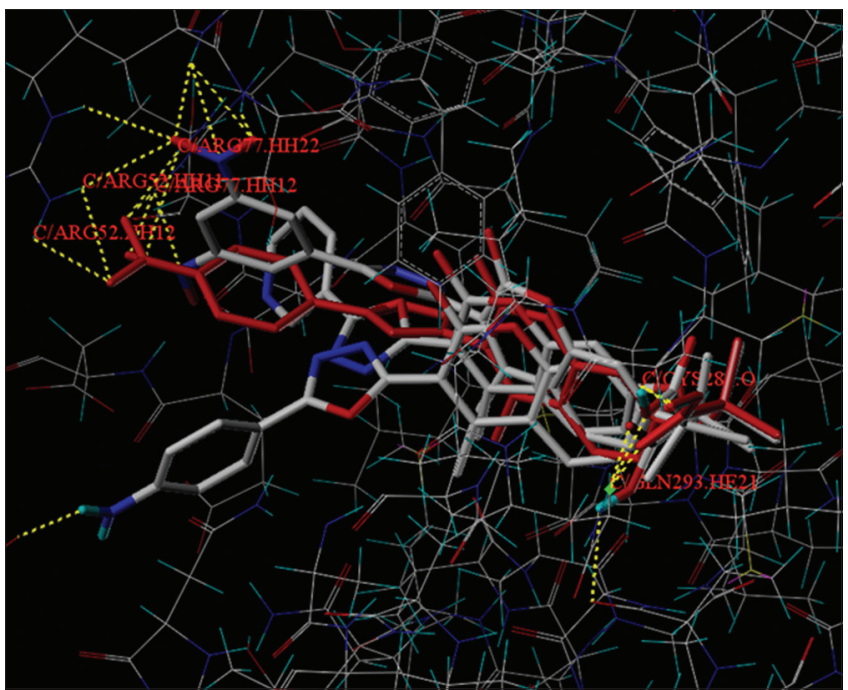

Fig. 11: Docking of 6 series with 3WCM.pdb

The docking study disclosed that the coumarin derivatives possessed high affinity in the directions 3WCM (Fig. 9). The docking score of coumarin derivatives $5 a-5 j$ and $6 a-6 j$ toward $3 W C M$ is shown in Table 2. Among the derivatives, compounds $6 \mathrm{f}$ and $6 \mathrm{e}$ displayed a good docking score of 8.8778 and $7.6619 \mathrm{Kcal} / \mathrm{M}$, respectively, show a better interaction with the protein squalene synthase, while the other coumarin derivatives exhibited a considerable score ranging from 3.8219 to $7.6015 \mathrm{Kcal} / \mathrm{M}$ (docking of 5 and 6 series with 3WCM. pdb is shown in Figs. 10 and 11, respectively, and also, $6 \mathrm{~g}$ reference is shown in Fig. 12). 
Table 1: Library of compounds

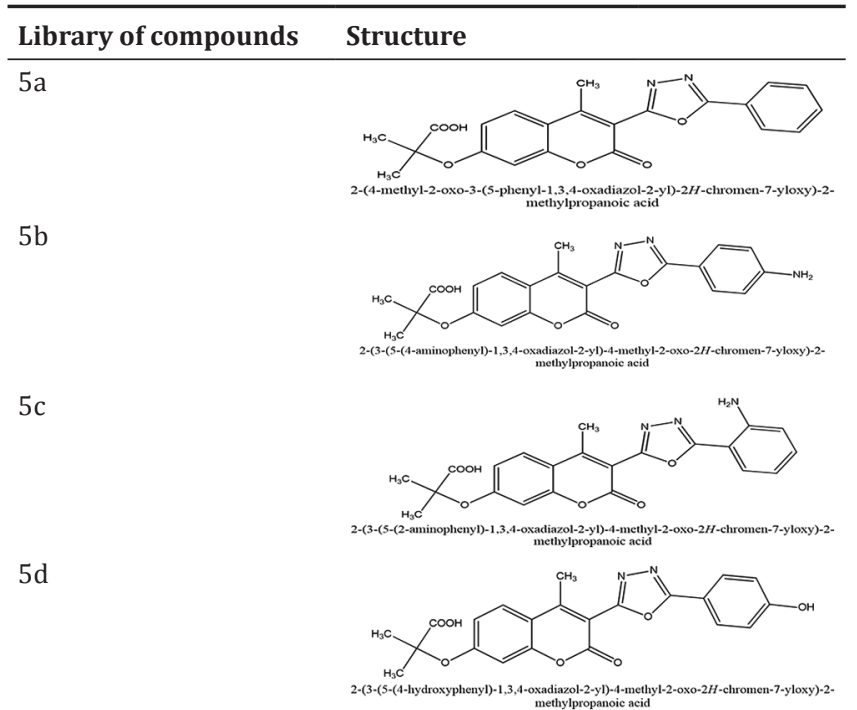

$5 \mathrm{e}$

$5 f$

$5 g$

$5 \mathrm{~h}$
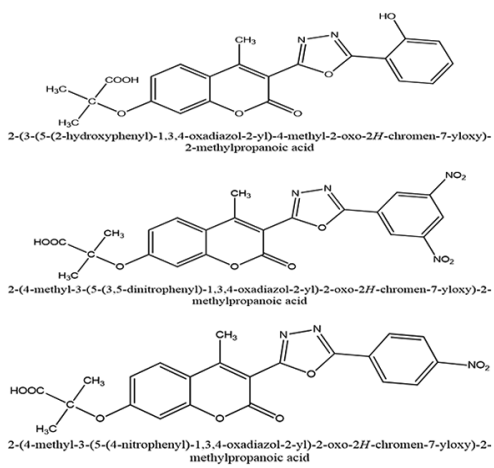

$5 \mathrm{i}$

$5 j$

$6 a$

$6 b$
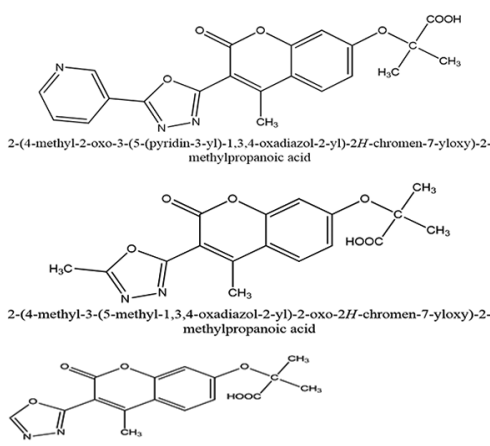

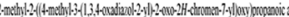

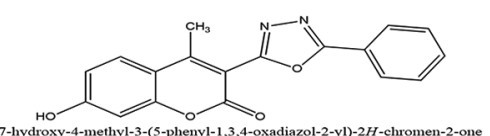

$6 c$

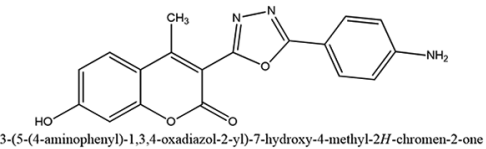

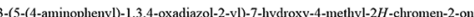

$6 \mathrm{~d}$
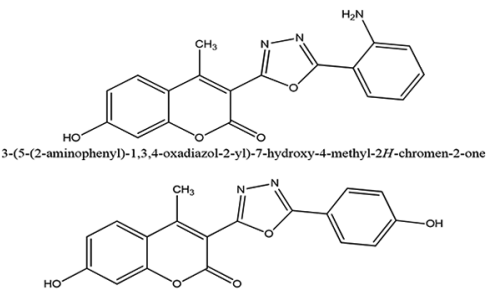

7-hydroxy-3-(5-4-4-hydroxyphenyl)-1,3,4-0xadiazol-2-y1)-4-methyl-2H-chromen-2-one

(Contd...)
Table 1: Continued...
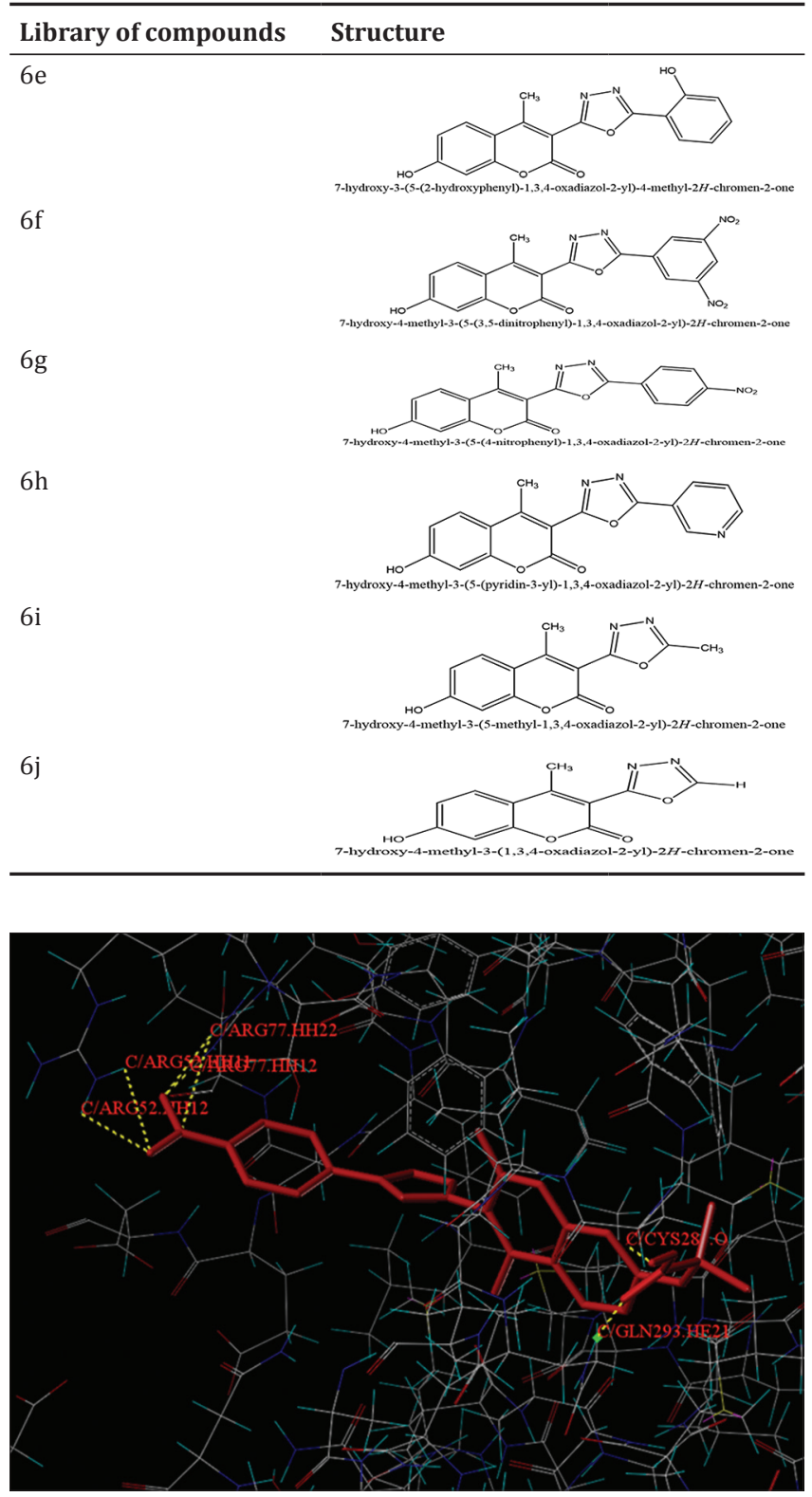

Fig. 12: $6 \mathrm{~g}$ reference

Molecular docking studies on squalene synthase (3WCJ)

The study of the designed substituted coumarin onto the active site of squalene synthase (PDB: $3 \mathrm{WCJ}$ ) was encouraging (Fig. 13). The cocrystallized ligand, E5S (3R)-3-(\{2-benzyl-6-[(3R,4S)-3-hydroxy-4methoxypyrrolidin-1-yl] pyridin-3-yl\} ethynyl) - 1-azabicyclo [2.2.2] octan-3-ol, showed a score of $7.4301 \mathrm{cal} / \mathrm{M}$ with the interactions with the all-around residues Phe54A, Phe288A, Leu183A, Leu76A, Val179A, Val175A, Tyr73A, Gln293A, Cys 289A, Tyr73A, and Leu211A strongly contributed to the stabilization.

The study displayed that the coumarin derivatives possessed high affinity in the direction of 3WCJ (Fig. 14). The docking score of coumarin derivatives $5 a-5 j$ and $6 a-6 j$ toward $3 W C J$ is shown in Table 3. Among the derivatives, compound $6 \mathrm{~g}$, 6f, and $6 \mathrm{~d}$ showed a good docking score of $8.355,7.9677$, and $7.5437 \mathrm{Kcal} / \mathrm{M}$, respectively, greater than that of the cocrystallized ligand $3 \mathrm{WCJ}$, indicating a greater interaction with the protein squalene synthase and spotlighting its capability to prevent the same and create a remarkable antidyslipidemia action, while the other coumarin derivatives showed a considerable score ranging from 4.0224 to $7.3892 \mathrm{Kcal} / \mathrm{M}$. The 
docking of 5 and 6 series with 3WCJ.pdb is shown in Figs. 15 and 16, respectively.

Molecular docking studies on squalene synthase (3Q2Z)

The study of the designed substituted coumarin onto the active site of the enzyme squalene synthase (PDB: 3Q2Z) was encouraging (Fig. 17). The cocrystallized ligand, D9A, N-\{[(3R,5S)-7-chloro-5-(2,3dimethoxyphenyl)-1-(2,2-dimethylpropyl)-2-oxo-1,2,3,5-tetrahydro4,1-benzoxazepin-3-yl]acetyl\}-L-aspartic acid, shows a score after docking, i.e.,7.7031 cal/M with the influence with the all-around the residues Val179A, Ala176A, Phen288A, Leu183A, Pro292A, and Phen288A strongly contributed to the stabilization.

Conformationally analyzed and energy minimized structures aligned against common atoms interacting with protomol $3 \mathrm{Q} 2 \mathrm{Z}$ are shown

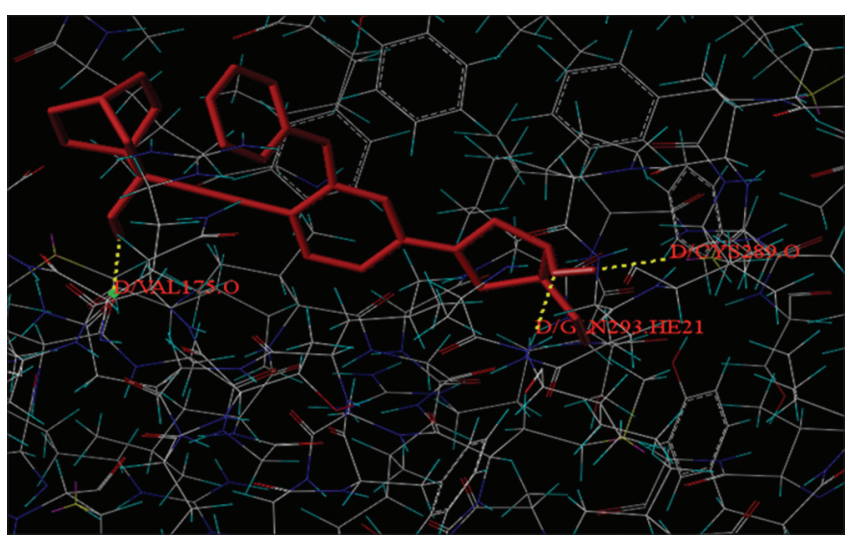

Fig. 13: Active binding in 3WCJ

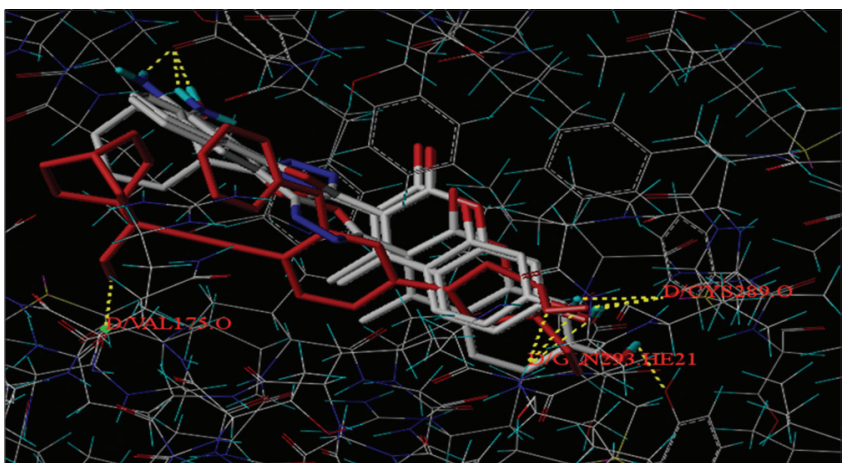

Fig. 14: Conformationally analysed and energy minimized structures aligned against atoms interacting with protomol $3 \mathrm{WC}$,

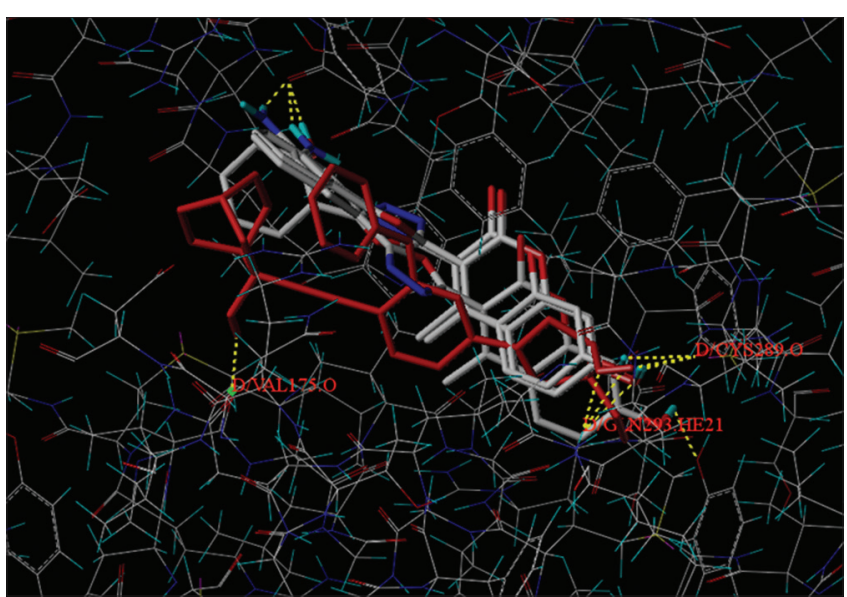

Fig. 15: Docking of 5 series with 3WCJ.pdb in Fig. 18. Docking score of coumarin derivatives $5 a-5 j$ and $6 a-6 j$ toward $3 \mathrm{Q} 2 \mathrm{Z}$ is shown in Table 4 . The study disclosed that, among the derivatives, compound $5 \mathrm{~d}$ showed a better score of $8.0496 \mathrm{Kcal} / \mathrm{M}$, respectively, greater than that of the cocrystallized ligand $3 \mathrm{Q} 2 \mathrm{Z}$ which shows a great interaction with the protein squalene synthase and draws special attention to its ability to inhibit the same and creates an important anti-dyslipidemic action, while the other coumarin derivatives showed a considerable docking score extend between 3.924 and $7.5023 \mathrm{Kcal} / \mathrm{M}$. The docking of 5 and series with 3Q2Z.pdb is shown in Figs. 19 and 20, respectively.

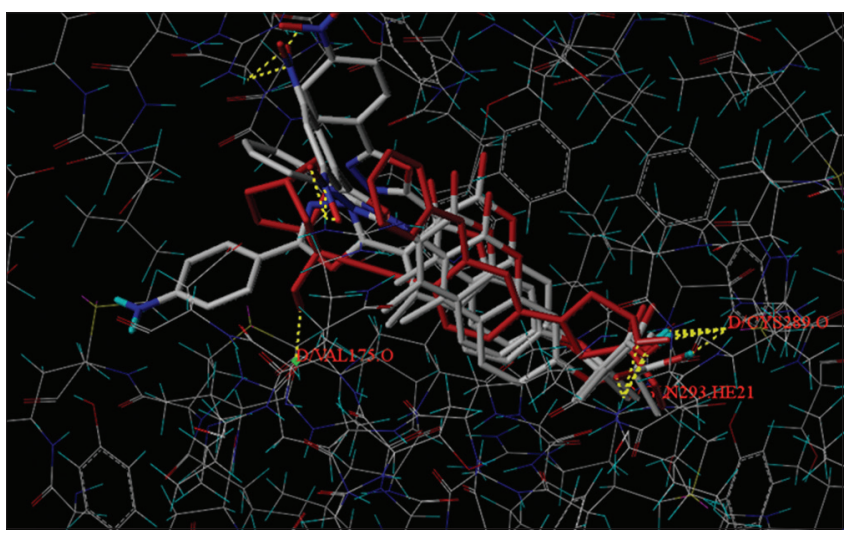

Fig. 16: Docking of 6 series with 3WCJ.pdb

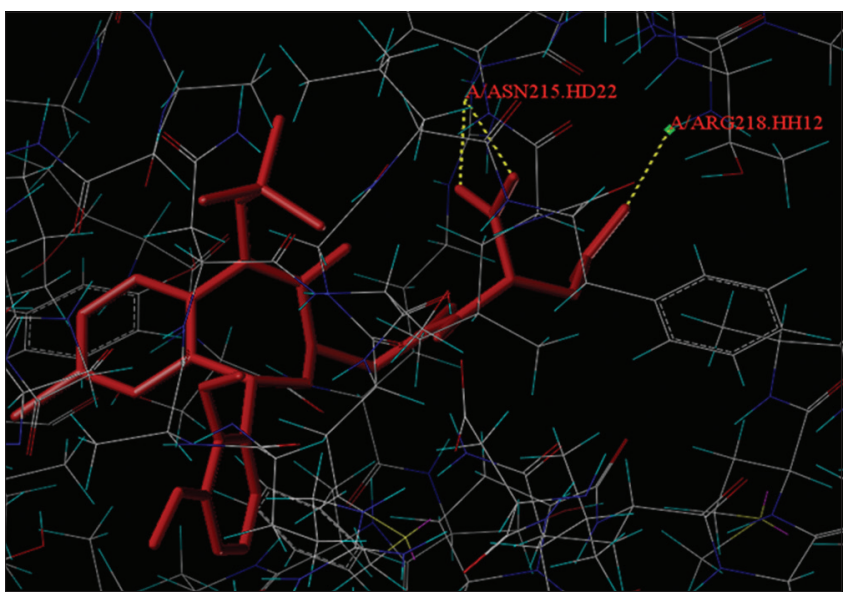

Fig. 17: Active binding in 3Q2Z

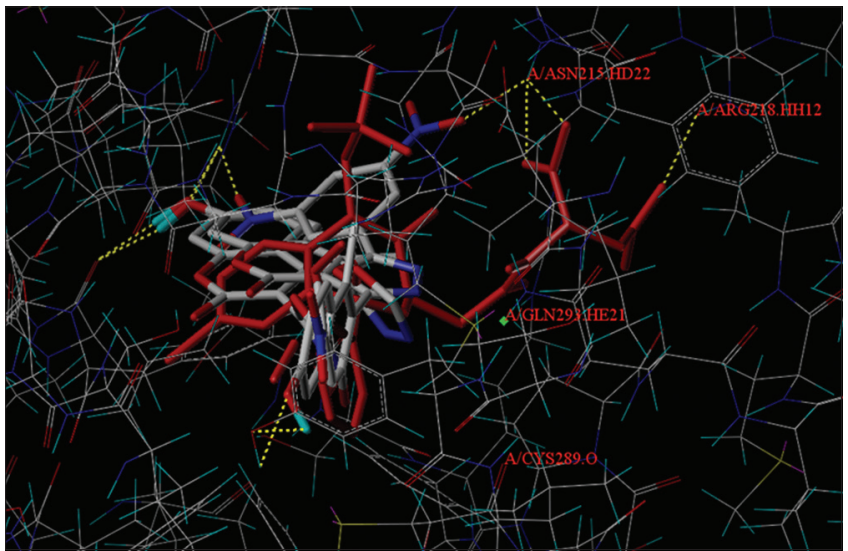

Fig. 18: Conformationally analyzed and energy minimized structures aligned against common atoms interacting with protomol 3Q2Z 
Table 2: Docking score of coumarin derivatives $5 \mathbf{a}-5 \mathbf{j}$ and $6 \mathbf{6}-\mathbf{6 j}$ toward 3WCM

\begin{tabular}{llll}
\hline Name & Total score & Crash & Polar \\
\hline 3WCM & 10.0858 & -1.0032 & 0 \\
5a & 6.0303 & -0.9745 & 0.6693 \\
5b & 6.2748 & -1.0513 & 0.8472 \\
5c & 6.4401 & -0.9485 & 1.5438 \\
5d & 6.7267 & -1.4343 & 1.1061 \\
5e & 6.7664 & -2.0112 & 1.9912 \\
5f & 6.0301 & -1.874 & 3.6454 \\
5g & 6.1686 & -0.9854 & 2.6787 \\
5h & 5.7272 & -0.9888 & 0.7757 \\
5i & 4.6311 & -0.9516 & 0.7607 \\
5j & 3.8219 & -0.8645 & 0.9766 \\
6a & 6.8195 & -1.9221 & 0.9764 \\
6b & 7.4204 & -1.906 & 2.3131 \\
6c & 7.6015 & -1.4632 & 1.0385 \\
6d & 7.3004 & -3.1822 & 4.9339 \\
6e & 7.6619 & -1.6783 & 1.0828 \\
6f & 8.8778 & -2.8572 & 4.8447 \\
6g & 6.9703 & -3.1642 & 3.4574 \\
6h & 6.6916 & -2.8632 & 2.2597 \\
6i & 6.1307 & -2.21 & 2.3035 \\
6j & 5.8351 & -1.7756 & 3.0013 \\
\hline
\end{tabular}

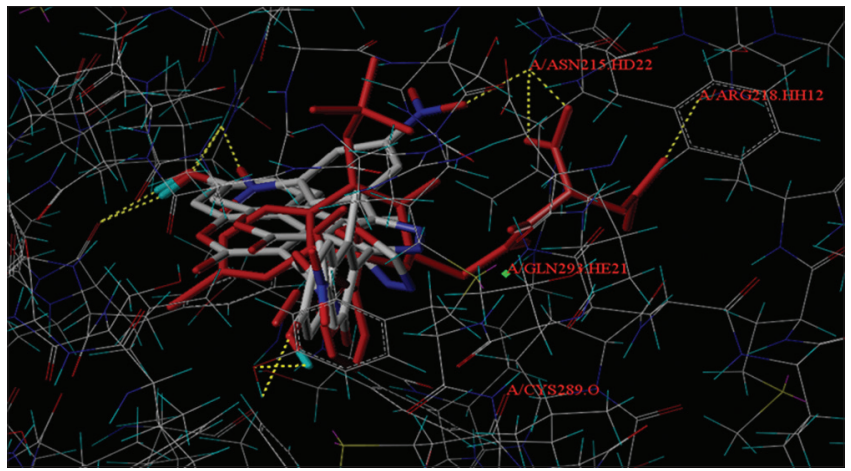

Fig. 19: Docking of 5 series with 3Q2Z.pdb

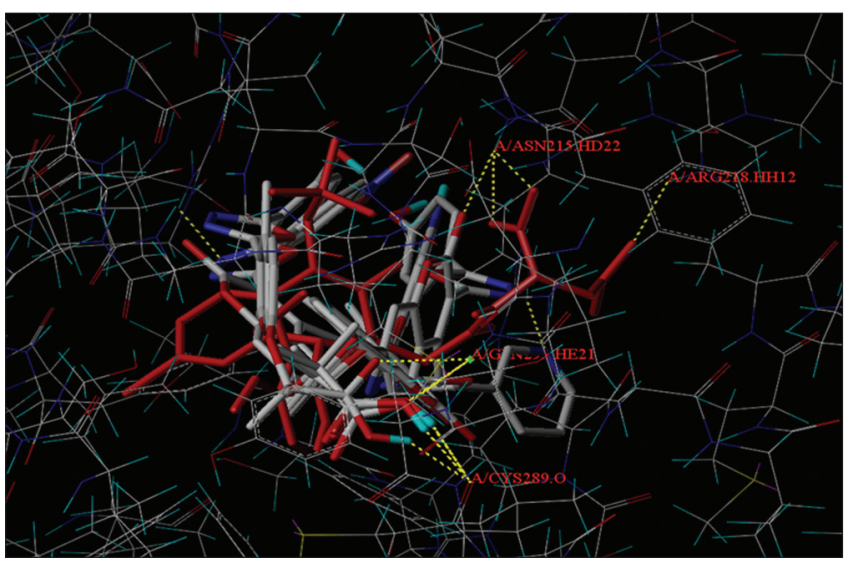

Fig. 20: Docking of 6 series with 3Q2Z.pdb

\section{CONCLUSION}

With the help of virtual evaluation, we have elaborated fast synthetically accessible coumarin-based compounds, and it is an advanced and original scaffold in the area of probable human squalene synthase inhibitors. Especially, we have designed a group of compounds from a common scaffold having various groups attached to the coumarin ring, by considering the both the compatibility with ligand binding domain and synthetic accessibility of squalene synthase. Some of the developed
Table 3: Docking score of coumarin derivatives $5 a-5 j$ and $6 a-6 j$ toward 3WCJ

\begin{tabular}{llll}
\hline Name & Total score & Crash & Polar \\
\hline 3WCJ & 7.4301 & -1.8869 & 3.0632 \\
5A & 5.7669 & -1.0303 & 0.1536 \\
5B & 7.0856 & -1.5897 & 0.9592 \\
5C & 6.7155 & -1.4323 & 2.0573 \\
5D & 6.5133 & -0.725 & 1.3759 \\
5E & 6.3168 & -1.5314 & 2.0522 \\
5F & 6.3762 & -1.2533 & 4.3744 \\
5G & 5.7789 & -1.2797 & 3.2303 \\
5H & 5.0926 & -0.967 & 0 \\
5I & 4.3926 & -0.5829 & 0.1326 \\
5J & 4.0224 & -0.8375 & 1.0627 \\
6A & 7.3178 & -3.0337 & 1.6263 \\
6B & 7.1507 & -2.9978 & 2.1212 \\
6C & 7.1805 & -4.5957 & 0.8395 \\
6D & 7.5437 & -4.5924 & 1.2254 \\
6E & 7.3892 & -1.6322 & 2.1942 \\
6F & 7.9677 & -2.3664 & 5.8064 \\
6G & 8.355 & -3.4986 & 5.3276 \\
6H & 6.8857 & -2.0305 & 2.0567 \\
6I & 6.3393 & -1.441 & 4.0459 \\
6J & 5.4802 & -0.8153 & 1.0872 \\
\hline
\end{tabular}

Table 4: Docking score of coumarin derivatives $5 a-5 j$ and $6 a-6 j$ toward 3Q2Z

\begin{tabular}{llll}
\hline Name & Total score & Crash & Polar \\
\hline 3Q2Z & 7.7031 & -1.8378 & 2.3055 \\
5a & 5.7563 & -0.6161 & 0.0008 \\
5b & 6.6484 & -1.0744 & 1.8029 \\
5c & 5.8023 & -0.8269 & 0.0118 \\
5d & 8.0496 & -1.2195 & 2.5386 \\
5e & 6.7664 & -2.0112 & 1.9912 \\
$5 f$ & 5.1324 & -0.7698 & 2.7422 \\
5g & 5.0874 & -2.1981 & 1.869 \\
$5 \mathrm{~h}$ & 6.1545 & -0.9184 & 2.1254 \\
$5 \mathrm{i}$ & 4.4302 & -1.6642 & 2.1496 \\
$5 \mathrm{j}$ & 3.6924 & -0.6029 & 2.0317 \\
6a & 6.2653 & -1.2328 & 1.9052 \\
6b & 6.2263 & -1.3705 & 1.8469 \\
6c & 6.9278 & -1.1291 & 1.494 \\
6d & 6.3276 & -1.0056 & 3.04 \\
6e & 6.4225 & -2.0735 & 1.3061 \\
6f & 6.5614 & -1.138 & 4.7483 \\
6g & 7.5023 & -1.7564 & 4.1302 \\
6h & 6.3419 & -1.9915 & 2.6184 \\
6i & 6.0045 & -1.213 & 1.2191 \\
6j & 5.0727 & -0.9799 & 1.714 \\
\hline
\end{tabular}

compounds show better binding property than ligand, and in 3q2Z (score: 7.7031), the compound $5 \mathrm{~d}$ shows better binding property than the protein with a score of 8.0496. Furthermore, $6 \mathrm{~g}$ and $6 \mathrm{c}$ have good binding property. In 3WCM (10.0858), the compound $6 \mathrm{f}$ has a better property with a score of 8.355 . In 3WCJ (7.4301), the compounds $6 \mathrm{~g}$ and $6 \mathrm{f}$ show better binding property than the protein with score of 8.355 and 7.9677 , respectively.

\section{ACKNOWLEDGMENTS}

The authors express their gratitude to the JSS Academy of Higher Education and Research and JSS College of Pharmacy, Mysuru, for providing necessary support in due course of the work.

\section{AUTHOR'S CONTRIBUTIONS}

Author is a faculty in the division of Pharmaceutics and Pharmaceutical Chemistry and the work contributed on faculty development program in the institution. 


\section{CONFLICTS OF INTEREST}

The authors confirm that this article content has no conflict of interest.

\section{REFERENCES}

1. Amin KM, Abou-Seri SM, Abdelnaby RM, Rateb HS, Khalil MA, Hussein MM. Synthesis and biological evaluation of novel coumarin derivatives as potential antimicrobials agents. Int J Pharm Pharm Sci 2016;8:109-16.

2. Jain KS, Kathiravan MK, Somani RS, Shishoo CJ. The biology and chemistry of hyperlipidemia. Bioorganic Med Chem 2007;15:4674-99.

3. Gordon T, Castelli WP, Hjortland MC, Kannel WB, Dawber TR. High density lipoprotein as a protective factor against coronary heart disease. The Framingham study. Am J Med 1977;62:707-14.

4. Reddy KS, Prabhakaran D, Chaturvedi V, Jeemon P, Thankappan KR, Ramakrishnan L, et al. Methods for establishing a surveillance system for cardiovascular diseases in Indian industrial populations. Bull World Health Organ 2006;84:461-9.

5. Halgren TA, Murphy RB, Friesner RA, Beard HS, Frye LL, Pollard WT, et al. Glide: A new approach for rapid, accurate docking and scoring. 2. Enrichment factors in database screening. J Med Chem 2004;47:1750-9.

6. Mi R, Bai XT, Tu B, Hu YJ. Unraveling the coptisine-ctDNA binding mechanism by multispectroscopic, electrochemical and molecular docking methods. RSC Adv 2015;5:47367-76.
7. Ke YY, Chen YC, Lin TH. Structure of the virus capsid protein VP1 of enterovirus 71 predicted by some homology modeling and molecular docking studies. J Comput Chem 2006;27:1556-70.

8. Oliaro-Bosso S, Taramino S, Viola F, Tagliapietra S, Ermondi G, Cravotto G, et al. Umbelliferone aminoalkyl derivatives as inhibitors of human oxidosqualene-lanosterol cyclase. J Enzyme Inhib Med Chem 2009;24:589-98.

9. Visegrády B, Than NG, Kilár F, Sümegi B, Than GN, Bohn H, et al. Homology modelling and molecular dynamics studies of human placental tissue protein 13 (galectin-13). Protein Eng 2001;14:875-80.

10. Bok K, Abente EJ, Realpe-Quintero M, Mitra T, Sosnovtsev SV, Kapikian AZ, et al. Evolutionary dynamics of GII. 4 noroviruses over a 34-year period. J Virol 2009;83:11890-901.

11. Jain AN. Surflex: Fully automatic flexible molecular docking using a molecular similarity-based search engine. J Med Chem 2003;46:499-511.

12. Variya B, Modi S, Savjani J, Patel S. In silico molecular docking and pharmacokinetic prediction of gallic acid derivatives as PPAR- $\gamma$ agonists. Int J Pharm Pharm Sci 2017;9:102-7.

13. Huang SY, Zou X. An iterative knowledge-based scoring function to predict protein-ligand interactions: I. Derivation of interaction potentials. J Comput Chem 2006;27:1866-75.

14. Venkatachalam CM, Jiang X, Oldfield T, Waldman M. Ligand fit: A novel method for the shape-directed rapid docking of ligands to protein active sites. J Mol Graph Model 2003;21:289-307. 